\title{
Alterstice
}

Revue internationale de la recherche interculturelle

International Journal of Intercultural Research

Revista International de la Investigacion Intercultural

\section{Discours et pratiques de la diversité face à la " norme " en contextes français et algérien : quelle place pour la pluralité linguistique des élèves dans l'éducation scolaire et le soin orthophonique?}

\section{Matthieu Marchadour}

Volume 7, numéro 2, 2017

Diversité(s) au coeur des politiques et des pratiques : entre l'Europe et l'Afrique

URI : https://id.erudit.org/iderudit/1052568ar

DOI : https://doi.org/10.7202/1052568ar

Aller au sommaire du numéro

Éditeur(s)

Alterstice

ISSN

1923-919X (numérique)

Découvrir la revue

Citer cet article

Marchadour, M. (2017). Discours et pratiques de la diversité face à la " norme » en contextes français et algérien : quelle place pour la pluralité linguistique des élèves dans l'éducation scolaire et le soin orthophonique ? Alterstice, 7(2), 37-53. https://doi.org/10.7202/1052568ar
Résumé de l'article

Cet article, issu d'un travail de doctorat, offre une réflexion sur le plurilinguisme dans les cadres scolaires français et algérien ainsi que dans les prises en charge orthophoniques. Nous analysons les discours et les réactions des professionnels de l'éducation face aux différentes langues des enfants, tantôt élèves, tantôt patients. Les questions principales soulevées sont celles de la norme, de l'étrangéisation de la langue de l'autre et de la traduction, et ce, dans le but de comprendre les enjeux à la fois linguistiques, éducatifs, sociaux et politiques qui contribuent aux processus d'inclusion ou d'exclusion des enfants immigrés et "non francophones ». Comment des institutions et des acteurs chargés de l'éducation et de « soin du langage " peuvent-elles prôner l'éducation et le soin des élèves « allophones » et réussir leur intégration socio-scolaire lorsque les milieux scolaires et de soins ne prennent en compte ni le parcours, ni le capital migratoire, ni les langues de ces élèves? 


\section{7}

ARTICLE THÉMATIQUE

\section{Discours et pratiques de la diversité face à la "norme " en contextes français et algérien : quelle place pour la pluralité linguistique des élèves dans l'éducation scolaire et le soin orthophonique?}

Matthieu Marchadour ${ }^{1}$

\section{Résumé}

Cet article, issu d'un travail de doctorat, offre une réflexion sur le plurilinguisme dans les cadres scolaires français et algérien ainsi que dans les prises en charge orthophoniques. Nous analysons les discours et les réactions des professionnels de l'éducation face aux différentes langues des enfants, tantôt élèves, tantôt patients. Les questions principales soulevées sont celles de la norme, de l'étrangéisation de la langue de l'autre et de la traduction, et ce, dans le but de comprendre les enjeux à la fois linguistiques, éducatifs, sociaux et politiques qui contribuent aux processus d'inclusion ou d'exclusion des enfants immigrés et " non francophones ". Comment des institutions et des acteurs chargés de l'éducation et de " soin du langage " peuvent-elles prôner l'éducation et le soin des élèves " allophones » et réussir leur intégration socio-scolaire lorsque les milieux scolaires et de soins ne prennent en compte ni le parcours, ni le capital migratoire, ni les langues de ces élèves ?

\section{Rattachement de l'auteur}

${ }^{1}$ Laboratoire PREFics EA 7469 (Pôle de recherche Francophonies, Interculturel, Communication, Sociolinguistique), Université de Rennes 2, France et Université d'Alger 2 Bouzaréah, Algérie

\section{Correspondance}

marchadourmatthieu@gmail.com

\section{Mots clés}

sociolinguistique, éducation, orthophonie, " allophones », plurilinguisme

\section{Pour citer cet article}

Marchadour, M (2017). Discours et pratiques de la diversité face à la " norme » en contextes français et algérien : quelle place pour la pluralité linguistique des élèves dans l'éducation scolaire et le soin orthophonique? Alterstice, $7(2), 37-54$. 


\section{Introduction}

Quelle place laissent l'éducation scolaire et le soin orthophonique à la fois à la singularité et à l'hétérogénéité, notamment linguistiques, des enfants? Comment les professionnels de ces domaines, en contexte français et en contexte algérien, se positionnent-ils et réagissent-ils face à la pluralité linguistique des enfants qu'ils éduquent et soignent? Vis-à-vis de " la » norme linguistique, quelle valeur donnent-ils à l' " écart » et au dissensus, qui sont à la fois des conditions et des stimulations du politique et du culturel (Rancière, 2000, p. 61)?

Au cœur des interdépendances entre langues et sociétés, toujours en mouvement, ou " covariances » (Bulot et Blanchet, 2013, p. 6), se pose la question des normes, sur laquelle nous nous pencherons ici, et vis-à-vis de laquelle la figure de l' "allophone " servira de base à notre réflexion ${ }^{1}$ : on parle d'allophone pour désigner "tout élève parlant une autre langue que le français" selon la dénomination utilisée dans l'Éducation nationale française depuis 2012 (Ministère de l'Éducation nationale, 2012), en d'autres termes pour désigner un enfant " autre " par la ou les langue(s) qu'il parle et surtout celle qu'il ne parle pas ou pas comme il faut, soit " le français ", entendu comme le français scolaire de France.

Dans cet article, on étudie l'école comme lieu d'éducation et l'orthophonie comme domaine de soin ou de "rééducation » dans les rapports de leurs acteurs au(x) norme(s) linguistiques, et ce, dans le cadre de la scolarité secondaire des enfants en France. Dans le cas français, c'est la figure de l'élève dit " allophone " qui nous servira de révélateur des normes et des attentes implicites de l'école et de l'Éducation nationale, ce terme posant grandement question (Armagnague-Roucher et Rigoni, 2016; Ebersold et Armagnague-Roucher, 2017; Razafi et Traisnel, 2017). On verra en quoi divers acteurs de l'institution, qui constitue un puissant relais de la nation (Vincent, 2013, p. 235), de ses croyances et de ses projets, font état de difficultés à composer avec la présence de ces enfants dits allophones, dont la dénomination englobe à la fois leur non-francophonie, leurs parcours migratoires (EANA, "élève allophone nouvellement arrivé "), ainsi que, dans le collège où nous avons enquêté à Rennes, leur absence de scolarisation antérieure (élève NSA, " non scolarisé antérieurement »). Ces enfants sont donc souvent triplement étrangers aux yeux d’un État-nation (Agamben, 1995, p. 31) qui fonde sur la naissance et la « maîtrise » de " la » langue nationale (Armagnague-Roucher et Rigoni, 2016, p. 344; Blanchet, 2016, p. 62) sa conception de la citoyenneté et de l'existence politique. II y a peu encore étrangers à la France, étrangers au français (celui de l'école française) et étrangers à l'école (française), ces enfants constituent une triple épreuve pour l'« orthodoxie » sociale et politique française (Sayad, 2014, p. 45), illustrant clairement les conceptions de ce que suppose et exige la nation en matière de loyauté linguistique et culturelle. Nous verrons quelles sont les difficultés exprimées par ces acteurs du système éducatif français ainsi que par les thérapeutes du langage que sont les orthophonistes quant à l'accueil de ces enfants "allophones ". C'est précisément par l'intermédiaire des discours "ordinaires" de ces professionnels sur des enfants souvent perçus et désignés comme « extraordinaires » que nous pourrons avoir une idée de ce que permettent ou empêchent certains cadres professionnels quant à la prise en considération de ces enfants en tant que sujets, et sujets nécessairement pluriels (Fleury, 2015), notamment du point de vue linguistique. Au cœur de notre réflexion tiendront une place importante la question de la traduction en tant que mobilité et position « entre » (deux ou plusieurs langues) (Cassin, 2016, p. 231) et la question de la difficulté à faire appel, pour les orthophonistes françaises exerçant en libéral (Marchadour, 2018) comme pour l'institution scolaire (Rigoni, 2017), à des traducteurs ou des médiateurs culturels professionnels pour aider les familles qui parlent peu ou pas français. Ces faiblesses structurelles dans le dispositif d'accueil et d'accompagnement des immigrés constituent de ce fait une sérieuse atteinte à la dimension politique du soin et de l'éducation, comme à celle de toute médiation linguistique (Cattaneo et Scorzelli, 2017, p. 391).

Cette investigation sur les normes linguistiques dans l'éducation scolaire et le soin orthophonique en France sera éclairée par une "décentration" (une forme de translation) qui consistera à confronter les discours et les pratiques de professionnels français de l'éducation et du soin du langage avec ceux de leurs homologues algériens, afin de voir comment y sont envisagées les langues, le langage et le rapport aux normes et à l'hétérogénéité dans ces domaines. Nous postulons que la mise en perspective des discours et pratiques de ces acteurs (enseignants et

\footnotetext{
${ }^{1}$ Réfléchir (penser) et se voir réfléchi (avec l’autre).
} 
orthophonistes algériens) permettra d'apporter un éclairage stimulant sur les notions de normes et de langues, sur les conceptions du plurilinguisme et de la traduction entre les langues. Nous postulons également que cette analyse en contrepoint nous apportera des idées sur les dispositifs et pratiques que l'on pourrait envisager de mettre en place en France dans les domaines du langage, des langues, du soin et de l'éducation.

\section{Présentation de la recherche}

\section{Contexte et spécificités des terrains d'études}

Il convient de faire quelques rappels concernant les contextes spécifiques des deux terrains d'études auxquels nous faisons référence dans ce travail. La France est officiellement et constitutionnellement monolingue (Légifrance, 2018) alors que l'Algérie, avec la reconnaissance en 2016 dans la Constitution algérienne du tamazight comme langue " également nationale et officielle » (République algérienne, 2016) aux côtés de l'arabe (classique) officialise une part du plurilinguisme des citoyens algériens et de leurs pratiques quotidiennes. Ceci étant, outre la position toute particulière du français en Algérie et de "l'ambivalence profonde » de l'Algérie à l'égard de cette langue (Kebbas et Kara-Abbes, 2010, p. 14), l'arabe algérien, ou la dardja (arabe parlé quotidiennement par les Algériens), non seulement ne bénéficie d'aucune reconnaissance officielle, mais est même opposé à l'arabe classique ou littéraire. Lors de nos entretiens à Alger, une enseignante de français tiendra justement à bien s'assurer que nous étions au fait de cette distinction entre l'arabe scolaire et " littéraire ", et l'arabe spécifiquement algérien :

Les petits Algériens ne parlent pas arabe littéraire / quand ils rentrent à l'école primaire ils sont confrontés à une langue qu'ils ne connaissent pas [...] c'est l'arabe mais ce n'est pas leur langue / c'est la langue du pays / mais ce n'est pas leur langue maternelle / vous voyez $\uparrow(A 5,2016,17-21)^{2}$.

La formulation de cette enseignante est capitale pour saisir l'opposition entre les conceptions étatiques officielles et les réalités linguistiques quotidiennes : les enfants algériens sont scolarisés dans une langue qui, affirme-t-elle, "n'est pas leur langue ». C'est aussi ce que dénonce Taleb-lbrahimi, rappelant le rôle sanctionnant et culpabilisateur que peut avoir l'école par la promotion (officielle) de la langue arabe qui y est enseignée, éloignée de l'arabe algérien: "La langue de la famille est clairement mise en opposition avec celle de l'école et le rôle de cette dernière est de corriger la langue de l'enfant et par là même celle de la famille ! (Taleb-Ibrahimi, 2008, p. 141). Bien que l'État algérien reconnaisse officiellement une forme de plurilinguisme intra-national, on voit qu'il existe des oppositions et des hiérarchisations, érigées par l'école notamment, entre pratiques linguistiques familiales non légitimes et pratiques linguistiques scolaires légitimes.

En France, la situation des élèves dits allophones et les termes qui les définissent sont ceux d'un étranger, dans un contexte scolaire et national pensé comme monolingue. Cette situation et ces dénominations sont à entendre différemment de celles en vigueur au Canada, où ce même terme d'allophone recouvre d'autres problématiques. Comme le distingue Gohard-Radenkovic, "l'immigration est constitutive de la Constitution et donc de la société canadienne où l'intégration de l'individu est pensée dans une conception multiculturelle depuis longtemps » alors que « la Constitution française déclare la langue française comme seule langue officielle, constitutive de la cohésion nationale, et l'intégration de l'étranger se fait à travers l'apprentissage et la maîtrise d'une langue, celle de la nation " (Gohard-Radenkovic 2014, p. 7). C'est pourquoi, en France, les allophones, en tant qu'immigrés dans un État qui se définit comme monolingue, ne sont pas tant des "autres » reconnus et acceptés que des " autres" linguistiques aux yeux d'une institution qui les enjoint à devenir francophones avant tout. En somme, en France, que ce soit à l'école française ou dans des contextes de soin, " parler équivaut à parler français " (Rosenbaum, 2010, p. 37), et « ne pas parler français équivaut à ne pas parler du tout » (Varro, 1997, p. 87).

\footnotetext{
${ }^{2}$ Dans les extraits d'entretiens utilisés, F2 fait référence à un enseignant en France, le $\mathrm{n}^{\circ} 2$. FO2 fera référence à une orthophoniste en France, la $n^{\circ} 2$. A2, un enseignant en Algérie, le $n^{\circ} 2$, et AO2 une orthophoniste en Algérie, la $n^{\circ} 2.2016$ correspond à l'année durant laquelle a été enregistré l'entretien, et les chiffres correspondent aux lignes de l'entretien retranscrit, que l'on trouve en intégralité dans les annexes du doctorat (Marchandour, 2018).
} 
Dans une République dont la Constitution rappelle la primauté de la langue française, quelle signification donner exactement au terme allophone? Avant son usage officiel, Sayad notait déjà, à propos du terme «nonfrancophone ", une dangereuse performativité :

A-t-on seulement le droit de parler de "non-francophones"? On ne mesure jamais assez les conséquences de ces raccourcis lexicaux. Ce n'est pas seulement une affaire d'exactitude du vocabulaire. Ce vocabulaire a des effets réels sur l'école : il fait la chose qu'il nomme. Il suffit de parler de "non-francophones » [...] pour que les "non-francophones " existent à l'école, pour qu'on les perçoive comme tels. (Sayad, 2014, p. 101)

\section{Objectifs de recherche}

L'objectif de notre recherche est d'interroger les discours et les pratiques sur la diversité linguistique au sein de la société française, dans des cadres d'éducation scolaire et de soin orthophonique, et d'explorer les conceptions, souvent implicites, de ce que serait un « ordre » linguistique définissant une norme scolaire et nationale unique.

Pour réfléchir à ces notions de diversité, d'ordre et de norme, nous avons choisi d'étudier de près les discours et attitudes portant en France sur les enfants dits " allophones », dénomination qui nous indique qu'ils sont à la fois "autres " et " extraordinaires", soit dans le sens premier hors de l'ordinaire, hors de la norme, donc. C'est depuis cette conception de l'extraordinaire que nous interrogerons l'ordre linguistique qui domine les contextes que nous avons choisi d'étudier. Si ces enfants perçus comme "spécifiques " serviront de révélateurs des normes attendues et de la conception d'un enfant français "ordinaire " dans les situations françaises analysées, nous ferons également un "détour» par l'Algérie dans des situations professionnelles homologues (école et orthophonie) pour tenter de cerner les discours qui ont cours sur la diversité linguistique.

\section{Constitution des corpus}

Les extraits d'entretiens exposés ici sont issus de notre travail de doctorat (Marchadour, 2018), dans lequel nous avons mené 46 entretiens semi-directifs :

- 10 entretiens avec des enseignants (6 enseignants dits " ordinaires » et 1 enseignant spécialisé responsables des élèves "allophones ") et des membres du personnel (infirmière et psychologue) d'un collège français classé en Réseau d'éducation prioritaire $(R E P)^{3}$ dans lequel sont scolarisés des enfants dits « allophones NSA ».

- 13 entretiens avec 12 orthophonistes françaises (un entretien a été mené avec deux orthophonistes en même temps, FO5 et FO6, et un entretien a été mené deux fois, à un an d'écart, avec la même orthophoniste, FO3 et F013) à Rennes et dans sa proche périphérie, auprès d'orthophonistes exerçant en libéral,

- 13 entretiens en Algérie, plus précisément à Alger et dans la périphérie algéroise, avec 12 orthophonistes exerçant dans un cadre hospitalier ou en libéral, dont 2 entretiens avec la même orthophoniste (AO8 et AO13) à un an d'écart,

- 10 entretiens avec des enseignants d’un collège de la périphérie algéroise.

\section{Terrains d'étude et méthodologies}

Les enquêtes de terrain ont été mises en place par le biais de deux méthodes, l'observation participante ${ }^{4}$ et les entretiens semi-directifs. Préalablement à la réalisation des entretiens, j'ai effectué des périodes d'observation participante au sein d'une classe dite d'UPE2A (Unité pédagogique pour élèves allophones arrivants) NSA, à

\footnotetext{
${ }^{3}$ La politique d' " éducation prioritaire " a pour objectif de " corriger l'impact des inégalités sociales et économiques sur la réussite scolaire par un renforcement de l'action pédagogique et éducative dans les écoles et établissements des territoires qui rencontrent les plus grandes difficultés sociales », http://www.education.gouv.fr/cid187/l-education-prioritaire.html

4 J'emploierai dans cette partie intentionnellement le « je " plutôt que le «nous " scientifique pour indiquer que j'ai appris l'arabe maghrébin et que je connais le contexte pour y avoir séjourné : ce parcours personnel m'a permis d'être reçu par mes interlocuteurs en tant qu'observateur participant et locuteur arabophone, de mener ainsi à bien mon enquête mais aussi de nouer des liens qui ont grandement facilité les contacts et mon enquête.
} 
Rennes, et au sein de différents cours dans un collège privé de la périphérie algéroise. Si l'observation des séances d'orthophonistes n'a pas été possible en France, secret professionnel oblige, quelques orthophonistes algériennes m'ont proposé et permis d'assister à certaines de leurs séances de soin. Ces observations menées avant la mise en place des entretiens avec les orthophonistes m'ont permis d'affiner certaines questions prévues dans les entretiens, et d'en établir de nouvelles, notamment en notant les stratégies développées par mes interlocuteurs algérois.

La méthode de l'observation participante offrait de nombreux avantages dans le cas d'une étude de terrain, notamment de "réduire le paradoxe de l'enquêteur, de comparer les pratiques par rapport aux discours sur les pratiques, ainsi que d'enquêter de l'intérieur de l'interaction langagière et des "communautés linguistiques" étudiées " (Blanchet, 2012, p. 42). Cette posture a été possible grâce à la prise de contacts et l'établissement de bonnes relations avec les enseignants en France et en Algérie, ainsi qu'avec les orthophonistes en Algérie, qui m'ont accueilli et montré les spécificités de leur profession, dont des difficultés inhérentes à leurs conditions de travail. Si les échanges avec mes interlocuteurs algériens se sont déroulés majoritairement en français, ma connaissance, même relative, de l'arabe maghrébin et de l'arabe écrit m'ont été d'une aide précieuse. Cette connaissance de l'arabe maghrébin, et dans une moindre mesure de l'arabe algérois, associée à mon expérience préalable du Maghreb en général et de l'Algérie en particulier, m’a permis de ne pas être un étranger «total ». Ainsi, ma relative " aisance » à prendre contact et interagir avec ces différents professionnels a constitué, je pense, un moyen efficace d'observer certains phénomènes et de recueillir certaines informations, ce qu'un non-initié n'aurait pas pu saisir. Être à la fois étranger et familier des contextes étudiés m'a probablement prémuni contre un " engagement total » et sans distance. Cela rejoint la position de Blanchet lorsqu'il écrit : " Je ne crois pas qu'on puisse enquêter efficacement en étant exclusivement à l'intérieur de la communauté ni, à plus forte raison, exclusivement à l'extérieur » (2012, p. 44).

Notons que cette recherche ne prétend pas offrir une comparaison, au sens strict, entre deux systèmes institutionnels, l'un qui serait « le " système éducatif et rééducatif algérien et l'autre, «le " système français. Nous avons préféré parler de décentration et non de comparaison car il nous paraît ambitieux (et irréalisable) de vouloir dresser le tableau de ces deux contextes, l'un " algérien ", l'autre "français ». En observant des situations et écouté des témoignages sur des pratiques, nous avons constaté que les professionnels entremêlaient discours personnels et institutionnels, ou plutôt qu'ils avaient souvent fait leur le discour institutionnel attendu. Notre posture de décentration s'inspire de la conception de l' " écart " de Jullien, à savoir la mise en place non pas d'un rangement mais bien d'un " dérangement », qui fait apparaître non pas des identités, mais des " fécondités ", des " ressources » (Jullien, 2016, p. 41). Contrairement à une approche comparative qui soulignerait des différences en ayant recours à des descriptions, l'écart, lui, " engage une prospection » (p. 35-36), invitant à réfléchir sur des possibles, des autrement, du mobile et du mobilisable. Ce mobile et ce mobilisable, ce mouvement, cet energeia, œuvre en train de se faire, et pas ergon, travail fini, sont justement ce qui peut définir les langues (Cassin, 2013,; Gobard, 1976) comme les cultures (Jullien, 2016).

C'est pourquoi les enquêtes de terrain en Algérie, à Alger précisément (qui n'est pas non plus l'Algérie), ainsi que les propos des acteurs interrogés ont été des révélateurs de ce qu'il est possible de faire, de penser, mais aussi de refuser de faire, et de changer, notamment lorsque des cadres professionnels, au nom de la norme, œuvrent en fait contre ceux-mêmes qu'ils forment ou soignent, à savoir des êtres de facto pluriels (Fleury, 2015), des individus - ce à quoi nous invite l'orthophoniste Francine Rosenbaum, dont la réflexion sur les "soignants " s'étend à tous les professionnels qui ont affaire à l'humain :

Je pense que si nous, les soignants, avons le sentiment que les modèles qui régissent nos pratiques deviennent persécuteurs pour nos patients et ne nous permettent pas d'établir une solide alliance thérapeutique avec les parents des enfants multiculturels, cela signifie peut-être que le moment de mettre en discussion nos modes opératoires est arrivé. Dans un contexte gravement discriminant à l'égard des enfants de migrants, nous sommes amenés à nous poser la question de la trahison des théories et des pratiques professionnelles issues de l’histoire ethnocentrique de nos disciplines. (2011, p. 80) 


\section{Enseignants et orthophonistes français face aux enfants " allophones " : l'expression de difficultés et de manques}

\section{Enseignants français et élèves " allophones "}

En France, les entretiens ont été menés avec des enseignants "ordinaires " qui avaient des enfants étiquetés allophones NSA en "inclusion" (partielle) dans leurs classes. Plusieurs de ces enseignants ont fait part, lors des entretiens, de difficultés et de manques, notamment de formation et d'accompagnement. Cette expression d'un manque ou d'un besoin de formation des enseignants par rapport aux enfants définis comme allophones rejoint de récentes enquêtes (Rigoni, 2017) qui indiquent, comme dans nos entretiens, un cruel manque d'appuis, en particulier le besoin de traducteurs professionnels pour des familles ou enfants parlant peu ou pas du tout le français (Rigoni, 2017, p. 48), une absence de moyens, de temps et d'espace qui nuit à la prise en compte des plurilinguismes individuels. Voici quelques témoignages édifiants quant à la situation dans laquelle se trouvent certains enseignants :

Cette année j'ai découvert avec I'UPE2A NSA que du coup y'en a beaucoup qui n'ont pas été scolarisés et qui ont un niveau vraiment très faible et là c'est vraiment très compliqué au niveau de l'inclusion / ils peuvent pas suivre / du tout / ce qu'on fait en sixième ça va mais ceux qui arrivent en quatrième c'est très dur, quoi. (F2, 2017, p. 30-33).

L'usage systématique des adverbes "très » et "vraiment " pour renforcer l'expression des difficultés de cette enseignante de mathématiques, auxquels s'ajoute à propos de l'impossibilité de "suivre " le recours à la locution adverbiale totale " du tout ", font état d'une situation pour le moins insatisfaisante et de difficultés éprouvées par l'enseignante comme par les élèves. Une autre enseignante de mathématiques fera elle aussi état de I' « impossibilité » de la tâche qui lui est confiée :

J'ai beau essayer de leur faire faire des choses c'est très compliqué parce que comme ils ont pas été scolarisés antérieurement ils ont aucune autonomie / face à ce que je leur demande / et même les dessins géométriques pour eux c'est extrêmement compliqué / et même tenir une règle ils savent pas / il faut que je leur explique absolument tout / donc il faudrait que je sois tout le temps avec eux. (F6, 2017, p. 47-51)

Cet extrait de discours révèle la détresse et l'impuissance de cette enseignante, qui commence son propos par « j'ai beau ", ce qui indique la reconnaissance de son inefficacité, malgré ses efforts répétés. Ensuite, les formules "c'est très compliqué ", "c'est extrêmement compliqué » et les exemples qu'elle donne, comme "ils ont aucune autonomie ", "même tenir une règle ils savent pas " (le "même " désignant une action qu'elle estime relativement basique) ou " il faut que je leur explique absolument tout » et la conclusion " il faudrait que je sois avec eux tout le temps ", indiquent l'impossibilité de mener à bien sa tâche, ce qui donne une idée du poids que ressent cette enseignante, qui ne peut pas réaliser ce qu'elle " doit ", et a l'impression d'abandonner les enfants dont elle est responsable. "Abandonner ", c'est d'ailleurs précisément le terme qu'elle emploiera lorsque je lui demanderai comment elle vit cette situation d' « inclusion » de plusieurs élèves peu francophones dans des classes " ordinaires », du moins dans la sienne :

Pour moi c'est une mauvaise idée dans le sens où j'ai pas le temps de m'en occuper / y'a des fois où je prends le temps mais dans ces cas-là c'est au détriment de la classe / faut être honnête / et je pense qu'au contraire / enfin je me mets à leur place et je me dis / quand je suis un cours et que je ne comprends rien et que je suis censé comprendre ça me rend complètement dingue [...] pour moi ils s'ennuient [...] j'ai l'impression que je les abandonne en fait / je les abandonne la plupart. (F6, 2016, p. 59-69)

Lorsque, touché par sa description de la situation, je demande à cette enseignante si le processus d' « inclusion » de ces élèves " allophones » dans sa classe lui semble être un échec, ses propos traduisent la position intenable dans laquelle elle se trouve :

Ah oui pour moi oui / enfin en fait c'est dès que je les vois dans ma classe je me dis oh mais les pauvres ils comprennent rien / et là j'ai pas le temps / là je peux pas / parce qu'en particulier là où y'en a quatre c'est une classe qui a aucune autonomie de travail / et je suis obligée de m'occuper en gros de chacun / chacun veut en quelque sorte que je m'occupe de lui / donc ils ont aucune autonomie / je vais les voir chacun / et du coup c'est un peu [...] ouais je suis désespérée en les voyant / en 
me disant je ne peux toujours pas m'en occuper donc ils vont toujours pas progresser aujourd'hui quoi / et quelle est leur façon de voir l'école si on les laisse de côté dès qu'ils arrivent / je trouve ça complètement monstrueux en fait. (F6, 2017, p. 181-189).

Cette enseignante souligne à nouveau la défection et l'absence de l'institution pour des enfants qui nécessiteraient une attention, un temps et un lieu particuliers. Les nombreuses formes " oh mais les pauvres", "je suis désespérée ", "je trouve ça monstrueux " indiquent un grand désarroi mais aussi un sentiment de culpabilité. Ainsi, des expressions telles que "j'ai pas le temps ", " je peux pas ", " je ne peux toujours pas ", " on les laisse de côté dès qu'ils arrivent " illustrent tout le poids que porte cette enseignante face à ce que l'on peut qualifier de maltraitance institutionnalisée. Double maltraitance institutionnalisée, selon nous: celle de l'élève, qui est " abandonné ", mais aussi celle de l'enseignante, qui ne peut réaliser la double " mission » qui lui est confiée, à savoir enseigner et transmettre des savoirs et savoir-faire mais aussi prendre en charge ces enfants venus d'ailleurs. Maltraitance institutionnalisée pour ces deux acteurs, car c'est demander à une personne d'accomplir une tâche irréalisable. Plus loin, dans l'entretien avec une enseignante d'histoire-géographie, lorsque je reviens sur un sentiment de se trouver « démunie » face aux élèves « allophones », elle déclare :

Oui je me sens démunie / il y a des moments où je trouve que c'est compliqué / même si effectivement on peut utiliser des gestes / même si je me suis retrouvée en début d'année pour reprendre le cas de cet élève de troisième qui visiblement ne maîtrisait pas un mot de français / avec un livre de lecture / un album pour enfant / à essayer de lui expliquer ce qu'il avait sous les yeux / que ce soit le vocabulaire ou les images / et j'étais à la limite de ce dont j'étais capable / réellement / et je suis pas certaine d'avoir été très utile de ce point de vue-là pour lui / j'ai passé du temps avec lui / ça je pense que c'est toujours appréciable / mais pour autant je ne suis pas certaine de l'efficacité. (F1, 2017, p. 180-186)

"À la limite de ce dont j'étais capable » et "je ne suis pas sûre d'avoir été très utile » : on peut entendre un sentiment de révolte et de résignation lorsqu'elle analyse ses propres pratiques et déclare encore une fois qu'elle se sent esseulée, manquant de soutien pédagogique. Je lui demande si elle pense qu'un regard ou une aide extérieure (de l'établissement, de l'Éducation nationale ou d'ailleurs) aurait pu ou pourrait lui être utile, ce à quoi elle répond :

Moi je pense que le regard extérieur il est de toute façon toujours appréciable / parce qu'il y a tout un tas de choses qu'on fait sans se rendre compte qui ne sont pas des bonnes choses / parce que ça relève d'automatismes et que du coup le regard extérieur permet de corriger ces points-là et permet parfois / à deux cerveaux on est plus efficaces qu'à un seul. (F1, 2017, p. 190-193)

Elle confirme alors son besoin d'une personne extérieure à la classe, voire extérieure à l'Éducation nationale, qui apporterait de possibles améliorations à cette situation sans issue. Je lui demande quels manques elle a identifiés et quel type d'aide pourrait lui être bénéfique :

II manque cet accompagnement pas seulement linguistique mais plus général / moi je pense qu'on sous-évalue l'altérité / mais l'altérité de façon générale / c'est-à-dire qu'on se confronte à des façons de vivre en société qui sont différentes des nôtres et je pense que du coup les codes ne sont pas les mêmes / qu'ils ne disposent pas des nôtres mais que pour autant nous on n'a pas non plus les leurs / et que du coup je pense qu'il y a un moment où on a du mal à être efficace / à cause de cette altérité qu'on sait très très mal gérer / à l'Éducation nationale / mais pas que / j'imagine qu'ailleurs c'est pas forcément. (F1, 2017, p. 198-204)

Ce discours fait état d'un manque clair, selon elle, d'un " accompagnement " linguistique et culturel des enseignants comme des élèves. En déclarant que "l'altérité est sous-évaluée », elle pointe du doigt la difficulté des élèves désignés comme "allophones", ceux récemment immigrés en France (EANA) et ne parlant pas ou peu français, mais elle n'oublie pas non plus de rappeler que les enseignants eux-mêmes ne sont pas suffisamment formés pour comprendre les " codes » de certains enfants. En somme, cette enseignante remet en question les attentes ethnocentriques d'institutions françaises selon lesquelles ce serait à l'élève "allophone " et " nouvellement arrivé » de s'adapter à des normes scolaires et sociétales immuables, qui ne relèveraient pas du culturel et du mouvant, mais du naturel et de l'évident. Nous identifions ici un processus que Bourdieu (1982) qualifie de "naturalisation de la langue ", "à l'origine de son enfouissement dans des "allants de soi", de son oubli institutionnel voire de son déni collectif : une langue pensée en creux ou pire impensée " (Gohard-Radenkovic, 
2014, p. 25). Sous l'expression " on sous évalue l'altérité [...] de façon générale ", on peut entendre ce que notre interlocutrice expliquera plus tard dans l'entretien, à savoir la mise au jour de certains implicites culturels et normatifs, dont ceux du système scolaire français.

Je pense qu'il faudrait justement qu'il y ait le moins d'implicite possible / pour ces élèves-là pour leur faciliter la tâche au maximum / et là je suis pas sûre que ce soit vraiment ce qu'on fasse. (F1, 2017, p. 223-225)

Cette impression de devoir décoder les implicites scolaires, pour et avec ces enfants "étrangers " à l'école et à ses codes sociaux spécifiques, est une idée développée aussi par d'autres enseignants à propos des élèves " allophones":

Ils ont pas eu les codes du collège depuis la sixième quoi. (F5, 2017, p. 100-101)

II a pas en fait le mode d'emploi pour fonctionner dans les écoles. (F7, 2017, p. 194-195)

Je pense qu'on devrait encore plus travailler en équipe / je veux dire en équipe de maths mais aussi en équipe en général pour décortiquer ce que veulent dire / ce que veulent dire les mots des consignes [...] je pense qu'on devrait généraliser ça pour qu'il n'y ait plus cette difficulté arrivé en troisième quoi. (F6, 2017, p. 289-294)

Bah plus ça va plus je pense que / même pour des élèves francophones je pense qu'en termes de compréhension des textes de tout ça / je pense qu'on travaille pas assez le vocabulaire en classe / et quelles que soient les disciplines hein là je suis en train de / de me reposer plein de questions là-dessus / c'est mon nouveau champ de bataille / trouver des petits biais pour / je pense qu'il y a une lacune en termes de vocabulaire simple des fois / mais vraiment / et en plus en français c'est vraiment complexe parce que tu as un mot qui change de sens selon que tu es en français en SVT [sciences de la vie et de la Terre] / quand tu sors du collège. (F3, 2017, p. 214-220)

Puis de façon plus nette, cette dernière enseignante évoque la nécessité de " réduire ces implicites »:

Je pense que les collègues dans toutes les disciplines déjà prendraient le temps d'expliquer les mots dans leur discipline ce serait déjà énorme quoi / mais je pense qu'on s'en rend pas compte / parce qu'il n'y a pas de formation. (F3, 2017, p. 223225)

Ce manque et ce besoin de formation sont aussi évoqués par d'autres professionnels, qui décrivent une situation d'impréparation totale. Si certains enseignants semblent ne pas avoir ressenti les effets d'une absence de formation, d'autres en revanche l'expriment haut et fort. Lorsque je leur demande s'ils ont pu bénéficier d'une quelconque sensibilisation aux difficultés liées aux enfants « allophones", voici ce que des enseignants répondent:

Non / aucune / jamais / jamais / je fais sur le tas et dans l'improvisation la plus complète / non pas que ça m'intéresserait pas hein / mais la situation ne s'est pas présentée. (F1, 2017, p. 85-88)

Non / rien du tout / et cette année j'ai même pas été prévenue que j'allais avoir une classe UPE2A / je l'ai appris un peu par hasard / et je me suis sentie vraiment déstabilisée on m'avait pas prévenue avant / et au bout d'une semaine j'apprends / je vois apparaître une heure / UPE2A / je me dis zut c'est quoi ça / bon alors déjà sans formation j'ai dû aller leur assurer une heure de cours / bon après l'inclusion c'est différent mais / donc non sans préparation on est un peu comme ça sur le terrain / on essaie on / nous après on a des cours un peu généraux sur la différenciation / sauf que on n'a pas de problématique particulière liée au fait qu'ils ne parlent pas la langue / donc non / aucune formation. (F2, 2017, p. 138-145)

Non je trouve qu'on nous laisse vraiment nous débrouiller tout seuls. (F3, 2017, p. 112)

Ces propos témoignent de la déstabilisation que peuvent ressentir des enseignants de classes ordinaires face à des enfants désignés comme " extra-ordinaires ", du fait des écarts, notamment linguistiques, entre ce qu'ils possèdent et surtout ne possèdent pas par rapport à cet ordre scolaire. Cet " autre étranger ", perçu comme du " culturel », du " mobile ", du " mouvant », du "mobilisable ", de l' " autre éloigné " face à du " même identique ", met de facto en doute l' " évidence », le " naturel », le " normal ", voire le " normatif », le " stable » et l' "immuable ». Ce manque "cruel» (F1, 2016, p. 94) de préparation aux difficultées liées à ces écarts (Jullien, 2016), qui sont 
culturalisés et donc essentialisés, ainsi qu'à l'altérité que certains enseignants ont exprimé dans nos enquêtes ne constitue pas un cas isolé. Comme le remarque Rigoni dans une récente enquête, "La formation des enseignants aux problématiques migratoires et aux relations interculturelles est extrêmement faible voire inexistante, même pour ceux qui font le choix d'enseigner en UPE2A » (2017, p. 48). S'il paraît difficile de savoir si c'est grâce à la présence de ces enfants "allophones», de surcroît "NSA», que cette nécessité d'explicitation des "codes scolaires " apparaît fréquemment dans nos entretiens, un constat semble en revanche se confirmer : c'est que la présence de ces enfants triplement "étrangers " à l'école française entraîne, de nombreux travaux l'attestent (Ebersold et Armagnague, 2017; Nathan, 1995; Sayad, 2014), une véritable stimulation pour repenser la dimension politique de l'école, c'est-à-dire une école mouvante, surprenante et dissensuelle (Rancière, 2000, p. 61), dimension que certains chercheurs appellent à restaurer (Clément, Dreux, Vergne et Laval, 2012, p. 278).

\section{Orthophonistes françaises et enfants peu ou pas francophones}

Nos entretiens montrent que, face à des patients et des familles peu ou pas du tout francophones, récemment arrivées en France ou non, et face à la difficulté, notamment financière pour les orthophonistes en libéral, de faire appel à des traducteurs ou à des médiateurs interculturels, les professionnelles interrogées éprouvent d'énormes problèmes. Ces problèmes vont de l'accueil initial à l'explication aux patients et familles de ce que signifie le soin, puis la mise en place du soin lui-même, dans lequel le langage est évidemment central, problèmes amplifiés par le fait que les ressources linguistiques ne sont pas partagées par le soignant et le patient (Marchadour, 2018). Nous ne pouvons pas citer ici tous les témoignages des orthophonistes interrogées, mais nous fournissons un aperçu de leurs expériences face à des enfants plurilingues, non-francophones et récemment immigrés en France.

Le fait de recevoir des familles et des enfants qui parlent peu voire pas du tout français, sans avoir aucune aide extérieure pour pouvoir traduire ou expliquer aux familles les enjeux, le rythme et les conditions du soin orthophonique, est problématique. Plusieurs témoignages attestent de ces situations rencontrées :

Ce qui est compliqué c'est vraiment quand la famille ne parle pas français. (FO2, 2015, p. 14)

Parfois j'ai des parents qui ne comprennent pas ou quasiment pas le français / donc là c'est vraiment compliqué. (FO4, 2017, p. 128-129).

Mais alors donc avec les parents étrangers qui ne parlent pas la langue / ça devient compliqué / ça devient très très compliqué. (FO5, 2017, p. 46-48)

Parfois la communication est difficile mais ce n'est pas forcément que la langue / je trouve que le fait qu'ils appartiennent à une autre culture met une autre barrière en fait [...] je trouve que la barrière culturelle est plus forte que la barrière linguistique. (FO7, 2017, p. 14-19)

Ces obstacles amènent certaines orthophonistes qui travaillent en libéral, et donc en dehors d'une équipe et d'une structure qui disposent de moyens financiers plus importants, à refuser des familles ou des patients qui ne parlent pas ou ne parlent que trop peu le français. Ainsi, une orthophoniste dit à ce sujet :

Je suis vraiment stricte maintenant parce qu'il m'est arrivé de / vous avez un enfant qui ne parle pas français les parents qui ne parlent pas français / mais moi je suis complètement perdue [...] moi je peux pas / je peux pas travailler dans ces conditions. (FO2, 2015, p. 40-45)

L'orthophoniste déclare "être stricte maintenant", ce qui signifie qu'elle ne l'a pas toujours été, mais que le niveau de langue de l'enfant à soigner lui a posé trop de problèmes. La non-francophonie, ou l' " allophonie » des enfants, mais également la "francophonie exclusive " de l'orthophoniste, participent conjointement à cette exclusion du patient. Bien que ce témoignage soit le produit des faiblesses d'un système sociétal global et non d'une simple (mauvaise) volonté individuelle, il convient de noter que cette exclusion du soin pour raison

\footnotetext{
${ }^{5}$ Les orthophonistes interrogés lors de nos entretiens sont toutes des femmes.
} 
linguistique relève de l'article 225-1 du Code pénal français, et peut être taxée de discrimination ${ }^{6}$. Le fait que les orthophonistes interrogées ne réussissent pas à mettre en œuvre un soin de qualité, voire un soin tout court, dû à une compréhension insuffisante ou même une totale incompréhension des enjeux de familles peu ou pas francophones, pose un sérieux problème d'accès aux soins. Selon nous, ces obstacles d'ordre linguistique, loin de ne toucher que l'orthophonie, comme en témoignent d'autres professionnels (notamment Brisset et Leanza, 2015; Leanza, 2017; Mestre, 2017), ne relèvent pas uniquement de problèmes « techniques » mais posent la question de l'accès, par le biais de langues autres que le français, à des droits fondamentaux et, de ce fait, soulèvent une question politique et éthique majeure.

\section{Enseignants et orthophonistes algériens face au " plurilinguisme ordinaire " : quels discours sur la diversité et la variation linguistique?}

\section{Enseignants algériens et pratiques linguistiques ordinaires}

Les enquêtes auprès des enseignants algériens ont permis de révéler deux grandes constantes en matière de rapports aux langues et au plurilinguisme. Si certains enseignants déclarent utiliser toutes les langues ou variétés linguistiques qu'ils connaissent et jugées aptes à "faire passer le message ${ }^{7}$ à leurs élèves, d'autres en revanche semblent afficher une hostilité à l'égard de tout "mélange " de langues au sein d'un même cours. Nous nous concentrerons ici sur les témoignages des enseignants ayant une conception positive du recours à différentes langues en cas de besoin. Voici les réponses de quelques-uns de nos interlocuteurs sur les langues qu'ils emploient en cours :

English chiefly / but sometimes i swap between english / french / arabic / sixty english / thirty french and from time to time ten percent arabic / why / because we have pupils that do not master neither english nor french / so i have to talk in their language sometimes / to deliver the message [...] sometimes you need more words / extra words to explain the rules of grammar / or vocabulary / i can explain in French [...] and complete it in arabic yes / because the arabic is their native language / it helps. (A1, 2016, p. 79-90 $\left.{ }^{8}\right)$

Le plus c'est l'anglais [...] je parle anglais et quand vraiment ils bloquent ou / on va vers le français / mais beaucoup plus on explique en anglais. (A2, 2016, p. 11-14)

Si je leur parle je leur parle en dardja / quand je parle de techniques c'est la majorité du temps en français / des mots disciplinaires c'est beaucoup plus en arabe / parce que ça a une relation avec le / la religion [...] si par exemple tu dis à quelqu'un « ma t7echchemch » [...] « a7chem » / c'est pas comme tu dis « t'as pas honte » [...] ça fait rien [...] « e7chem » / tu dis « e7chem »/ tout de suite il se calme. (A4, 2016, p. 91-109) ${ }^{9}$.

J'essaie de / de faire une petite culture politique quand même pour les préparer / c'est des enfants qui sont amenés à vivre ensemble / dans le même pays / parler le français l'arabe / et je leur dis toujours ça c'est mon leitmotiv / quelle que soit la langue qu'on pratique l'important c'est de savoir ce qu'on fait avec. (A5, 2016, p. 531-534)

Moi je fais même pas attention quand je passe d'une langue à l'autre / déjà je ne fais même pas attention / je parle / mon idée suit en français puis je reviens en arabe / donc pour moi c'est / c'est une seule langue / voyez $\uparrow /$ et le message passe bien. (A8, 2016 , p. 131-135)

\footnotetext{
${ }^{6}$ « Constitue une discrimination toute distinction opérée entre les personnes physiques sur le fondement [...] de leur capacité à s'exprimer dans une langue autre que le français », Code pénal, article 225-1 (Légifrance, 2016).

${ }^{7}$ Un terme qui revient souvent dans les entretiens.

8 « Anglais principalement / mais parfois je jongle entre anglais / français / arabe / soixante (pour cent) anglais trente français et de temps en temps dix pour cent arabe / pourquoi / parce que on a des élèves qui ne maîtrisent ni l'anglais ni le français / donc je dois parler dans leur langue parfois / pour transmettre le message [...] parfois on a besoin de plus de mots / des mots supplémentaires pour expliquer les règles de grammaire / ou le vocabulaire / je peux expliquer en français et compléter en arabe oui / parce que l'arabe c'est leur langue maternelle / ça aide ». [traduction de l'auteur]

${ }^{9}$ On voit que la nomination même des langues est importante et délicate : il nomme ici la dardja, le français et l'arabe, mais parfois la dardja est nommée simplement " arabe » dans le discours des locuteurs, ou encore il arrive que l'on ne sache plus précisément si quelqu'un utilisant le terme « arabe » parle de la langue arabe classique ou de la dardja.
} 
Certains enseignants semblent insister sur l'importance et la priorité du fond sur la forme, ce que plusieurs d'entre eux désignent par " message » (A1, 2016, p. 82; A4, 2016, p. 279-280), et ce qui est aussi formulé différemment par l'enseignante de français précédemment citée : pour elle, l'important avec la langue est " ce que l'on fait avec " $(A 5,2016$, p. 531). Dans ces quatre cas, les " grilles » et les variétés linguistiques ne semblent pas avoir raison du " message " ni devoir faire perdre l'idée d'échange, de transmission, de "passage ». Une enseignante va même jusqu'à dire que le "mélange » de trois langues indépendamment identifiées par ses élèves constitue, à ses yeux, une seule et même langue, tout comme l'artiste algérien Fellag déclare que l'arabe, le français et le kabyle mélangés sont « sa vraie langue », « sa langue » (Caubet, 2004, p. 14-15).

En recourant au français et à l'arabe algérien pour une même formule, l'enseignant d'éducation physique et sportive (EPS) (A4) précédemment mentionné montre, d'une part, la cohérence de l'utilisation de certains registres linguistiques pour dire certaines choses et, d'autre part, l'inadéquation à utiliser le français pour exprimer certaines choses, de même qu'il est parfois difficile ou impossible de dire d'autres choses autrement qu'en français (Fellag, 1995, p. 37). C'est donc la pertinence des langues utilisées de manière adaptée aux contextes qui est invoquée ici. Expliquer en différentes langues, avec des mots « supplémentaires » (A1, 2016, p. 79-90) auxquels on a recours, n'est-ce pas l'une des idées de la traduction que mettent en avant Ricœur, Cassin ou Jullien ? Cassin parle ainsi de la traduction comme d'un "modèle de l'entre » (2016, p. 231), d'un " savoir-faire avec les différences » (2017, p. 9) et d'une manière "d'inventer entre les langues » (2017, p. 12), quand Jullien l'envisage comme le fait non pas de revenir à du familier, mais de "sonder » la différence (2006 , p. 41-42), tandis que Ricœur mentionne, pour sa part, le mouvement auquel nous poussent « l'incommunicabilité entre idiomes et la traduction malgré tout » (2004, p. 51-52).

\section{Orthophonistes algériennes : discours sur les normes linguistiques}

Pour ce qui est des orthophonistes, les entretiens avec les professionnelles algériennes donnent à voir un rapport aux normes intéressant et inédit. La plupart des orthophonistes interviewées soulignent que ce sont les patients et leurs familles qui déterminent la ou les normes linguistiques à partir desquelles seront conçus les soins. Les orthophonistes algéroises (et donc citadines) parlent l'arabe algérien, le français, et à des degrés divers l'arabe " classique ${ }^{10}$ et le kabyle, parfois l'anglais. Après avoir observé quelques séances auprès de ces orthophonistes, nous avons souhaité savoir quelles langues elles utilisaient durant leurs consultations, à Alger, face à des patients qui parfois arrivent de très loin (souvent ruraux) et susceptibles de parler d'autres variétés linguistiques qu'elles. Nous leur avons demandé quelles sont alors leurs stratégies. Voici quelques extraits de leurs réponses :

C'est un travail pour que l'enfant s'adapte à son environnement / pas au mien / ce qui fait que je travaille avec sa langue. (AO1, 2016, p. 65-66)

Ça dépend des parents qu'est-ce qu'ils utilisent à la maison [...] la plupart du temps on pose la question pour les parents / par exemple les parents du Sud on leur dit qu'est-ce vous dites à la maison 个 / le mot exact / on est obligées de l'utiliser / pour l'enfant ça lui fait plus facile pour apprendre le mot // je pense [...] le mot exact la prochaine séance je l'utilise comme il l'utilise à la maison. (AO5, 2016, p. 115-126)

J'essaie de m'adapter [...] c'est moi qui m'adapte / non je leur change pas aux enfants / c'est moi qui m'adapte / et c'est très difficile. (AO7, 2016, p. 291-293)

À chaque fois je demande aux parents qu'est-ce que ça veut dire ça qu'est-ce que ça veut dire ça $\uparrow /$ et moi j'accepte. (AO11, 2015, p. 70-71)

Des fois il y a des familles il y a des enfants qui parlent dardja c'est-à-dire le dialecte ils disent balle parce qu'on dit balle mais y'a d'autres qui disent kora [...] et d'autres qui disent balo [...] on demande aux parents est-ce qu'il dit balo ou ballon à la maison. (AO6', 2016, p. 146-161)

La dernière orthophoniste citée, qui lors de l'entretien était accompagnée d'une de ses collègues, me proposa plusieurs exemples de variantes lexicales pour désigner un même objet illustré sur une carte montrée à un enfant,

\footnotetext{
${ }^{10}$ C'est le terme que les personnes interrogées utilisent.
} 
en insistant sur le fait que ce sont à elles de s'adapter aux habitudes linguistiques des enfants et familles, et pas à ces derniers de se conformer aux normes pratiquées par les professionnelles. Elles donneront en exemple la tomate, dont la désignation varie selon les familles et les origines géographiques des individus :

Déjà ballon c'est rien du tout y'a des mots qui sont vraiment différents / par exemple la tomate en français. (AO6, 2016, p. 162-163)

Ici on dit tomâTech ${ }^{11}$ (AO6', 2016, p. 164)

À Biskra Tammich ${ }^{12} /$ euh sinon maTicha ${ }^{13}$ (AO6, 2016, p. 167)

TmâTem $^{14}$ (AO6', 2016, p. 168)

Il y a plusieurs donc on suit le patient [...] oui / on demande ce qu'il dit. (AO6, 2016, p. 169-171)

Ces deux orthophonistes se complètent pour me donner des exemples d'hétérogénéité linguistique " ordinaire ", en m'exposant cinq formes de dire, d'entendre et de voir la tomate. Elles concluent par une déclaration aussi laconique que puissante : "On suit le patient, on demande ce qu'il dit", rappelant que ce sont les patients qui fixent ici les usages linguistiques, et qu'elles prennent soin (le terme leur convient ici parfaitement) de partir du bagage linguistique de ces derniers. Pour poursuivre sur cet exemple, elles m'exposent un autre cas de figure :

Comme la dame a dit hier par exemple / pour la fleur moi je dis généralement warda ${ }^{15}$ / mais sinon la patiente elle a dit azzhâr $^{16}$ / oula zahra ${ }^{17} /$ mais bon zahra c'est // oula nouwwâr ${ }^{18}$ [...] mais on les comprend tous. (AO6, 2016, p. 173-181)

Là encore, au moins cinq mots différents sont mobilisés pour une même " image » ou une même référence. Ayant observé des séances durant lesquelles elles utilisent un imagier, je pointe du doigt une autre carte, et leur demande quels mots utilisent les enfants pour cette image :

Soit pouppoune / poupon / pouppoune / ou doubb ${ }^{19}$ / doubb c'est l'ours (AO6, 2016, p. 183)

Je leur demande alors si pour chaque carte il y a souvent autant de possibilités :

Déjà sûrement il y a deux c'est pour le français et l'arabe / sinon parfois en arabe il y a beaucoup de dialectes / il y a plusieurs dialectes. (AO6, 2016, p. 188-189)

Le minimum possible est "deux ", déclare cette orthophoniste. L'univocité de la réponse n'est donc pas envisageable, et l'on sait grâce à des auteurs comme Ricœur (2004), Cassin (2016) ou Jullien (2016) à quel point c'est justement cette impossible univocité, cette plurivocité inévitable commençant dès la dualitée ${ }^{20}$, qui rendent toute " ouverture ", tout " écart " possible (Jullien, 2016, p. 89), toute "vérité » relative et "compliquent l'universel » (Cassin, 2016, p. 25).

\footnotetext{
ثماطِّ 11

طمّيش 12

ماطيشة 13

تماطد 14

وردة 15

الز هار 16

ز هرة 17

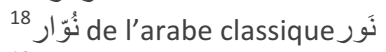

19 دُوَّب 18

20 " "Plus d’une langue" et "une langue, ça n'appartient pas", voilà nos deux mots d'ordre pour penser la traduction » (Cassin, 2016, p. 220).
} 
Non seulement ces orthophonistes " acceptent " ou valident toutes les variétés utilisables pour un même mot, mais elles communiquent aux familles la liste des mots qui vont être travaillés en séance, pour que les parents puissent s'y préparer et fournir les formes qu'ils utilisent. L'une des deux poursuit :

Ils traduisent oui / ils sont pas obligés de me suivre [...] bien sûr / c'est à leur convenance [elle sourit] [...] ils suivent leur(s) langue(s) leur(s) dialecte(s) si on peut dire / ils ont raison / ils vont pas parler comme moi. (AO6, 2016, p. 197-203)

Par exemple moi je suis de A [une ville à 150 kilomètres à l'ouest d'Alger] / et j'habite là sur Alger / il y a des mots c'est pas les mêmes / j'essaie de les utiliser selon les malades / je change les mots [...] il y a aucun problème / j'accepte / j'accepte / c'est tout à fait normal ils vont pas me suivre / ce serait illogique [elle rit doucement] il va pas vivre avec moi. (AO6, 2016, p. 204-210)

Là encore, le discours de l'orthophoniste est clair : elle soigne l'enfant et son langage en rapport et en cohérence avec son contexte social et pas celui d'une norme surplombante, nationale ou officielle unique. Pour mener à bien son travail, c'est elle, la professionnelle du langage, de la parole et de la communication, qui " consulte » les parents pour connaître leurs "valeurs linguistiques", c'est-à-dire ce qui fait sens pour eux, soit les variétés linguistiques qu'ils utilisent avec l'enfant qu'elle soigne. Quand je lui fais part de mon intérêt pour ses pratiques, elle me répond : "il va pas vivre avec moi », replaçant alors le patient dans son milieu et dans ses perspectives d'avenir. Ces professionnelles rappellent alors, par leur geste, le fait que le soin comporte, comme le langage, la culture et la politique, une part essentielle d'imprévu et d'incalculable (Jaffredo, 2016, p. 218). Comment, alors, ne pas voir dans ces pratiques et ces discours ce que Cassin appelle un "savoir-faire avec les différences " à propos de la traduction (Cassin, 2017, p. 9)? Ces pratiques "ordinaires », dont certaines orthophonistes semblent me rappeler la «banalité » (AO5, 2016, p. 186), pourraient relever de ce que nous choisissons d'appeler une " disponibilité à la translation ", soit des spécialistes qui connaissent la valeur et l'importance de l" " écart ", de la relativité et la mobilité des normes, dont Canguilhem a montré qu'elles étaient " polémiques et dynamiques " (1966, p. 176). Bien que ces pratiques professionnelles semblent relever, selon les acteurs interrogés, de plurilinguismes individuels ordinaires, dans le sens d'habituels, au sein de la société algérienne, elles pourraient aussi être, contrairement aux contextes français où le monolinguisme reste la norme, qualifiées d' " ouvertes " et d'attentives. Elles pourraient ainsi inspirer des changements, même minimes, sur les façons actuelles d'envisager la norme linguistique attendue par l'institution scolaire et intériorisée par les acteurs de la société française. Comment alors, face à ces pratiques algériennes " banales » de la prise en considération des normes linguistiques de l'autre, ne pas songer à cette vision de Jullien, qui écrivait il y a peu que le " monde à venir » devait être celui de l’ " entre-langues », donc de la traduction et pas du monolinguisme institutionnel $(2016$, p. 89)?

\section{Conclusion}

Nous ne pouvons que constater l'arrivée constante, en France (Ministère de l'éducation nationale, 2017), d'enfants et de jeunes ayant émigré, étant parfois accompagnés, parfois seuls, parlant parfois « le " français (celui reconnu comme tel par l'institution scolaire française), parfois « un » français, parlant souvent d'autres langues ou variantes que le français scolaire de France, "ayant des expériences scolaires aussi diverses que leurs expériences migratoires " (Rigoni, 2017, p. 49), mais tous désignés indistinctement sous le terme d' " allophones ", terme dont I'utilisation en contexte français soulève des interrogations (Armagnague-Roucher et Rigoni, 2016; Ebersold et Armagnague-Roucher, 2017; Razafi et Traisnel, 2017). Face à ces situations, il convient d'analyser attentivement les conditions d'accueil, d'évaluation, d'éducation et de soin réservées aux enfants et aux familles parlant d'autres langues que le français. Si l'on veut remettre en question une conception "glottophobe » de la société française et de I'Éducation nationale (Blanchet, 2016, p. 41), si l'on refuse que soient relégués au rang de "citoyens de deuxième catégorie qui ne peuvent bénéficier de leurs droits qu'en français » (Blanchet, 2016, p. 112) toutes les personnes qui parlent dans l'espace national français une ou d'autres langues que "le " français, il semble capital de veiller à ce que soient mis à disposition des interprètes, des traducteurs et des médiateurs soumis au secret professionnel. Recourir à ces professionnels de l'interprétariat permettrait de ne plus laisser aux familles le souci de s'organiser par elles-mêmes pour comprendre et se faire comprendre (Marchadour, 2018; Rigoni, 2017, p. 48), mais permettrait également aux patients de bénéficier d'un accès direct au soin, sans que des proches empiètent sur leur droit à l'intimité et au secret. Si nos entretiens (FO4, 2017, p. 215-223) comme certains travaux récents (Noël, 2015; Rosenbaum, 2012) montrent que les conditions d'accueil en orthophonie reposent implicitement sur 
une conception monolingue et monoculturelle de la société française (Rosenbaum, 2003, p. 411), et si d'autres publications attestent d'attitudes qui se sont rigidifiées dans l'espace scolaire français quant à la diversité linguistique (Blanchet, 2016), il est nécessaire de rester vigilant quant à la valeur et la place attribuées à la pluralité linguistique et aux personnes parlant d'autres langues que le français dans les contextes français. Si nous devons garder à l'esprit la spécificité de l'histoire de la constitution de l'État-nation français, il nous paraît toutefois crucial que les actions éducatives et thérapeutiques retrouvent leur dimension intrinsèque, c'est-à-dire composent avec « le pluriel et l'incirconscriptible » dont sont constituées les personnes en tant que sujets (Fleury, 2015, p. 110).

Les difficultés exprimées par les enseignants français " ordinaires " face aux élèves " extraordinaires » que sont les enfants désignés comme allophones, c'est-à-dire peu ou pas francophones, perçus "plurilingues ", récemment immigrés et parfois non scolarisés antérieurement, soulèvent des interrogations à la fois éducatives, sociales et politiques au sein des contextes dans lesquels ils sont " accueillis ". Cette perception des élèves comme " autres différents ", qui les accule à une étrangeté parfois radicale, se révèle dans des situations de soin orthophonique et d'éducation scolaire par le biais de la langue. Mais ces écarts linguistiques et culturels, réels ou fantasmés, ne doivent pas être interprétés comme de simples problèmes "techniques", sous peine d'en occulter toute la dimension politique (Sayad, 1999, p. 316). Triple dimension politique: de l'émigration-immigration, du plurilinguisme ${ }^{21}$ ou de l'alterlinguisme (par rapport à la langue nationale) et enfin de l'école en tant que lieu non neutre (Sayad, 2014, p. 160), c'est-à-dire lieu non seulement de formation, mais aussi et peut-être surtout lieu de conformité et de conformisation (2014, p. 162) à des normes linguistiques exigées par l'État-nation et la conception dont il relève. Face à des situations françaises où le plurilinguisme ou l' "alterlinguisme " (" allophone ", " autre " que «le » français) remettent sérieusement en question les cadres politiques et institutionnels (Rosenbaum, 2011, p. 70) dans lesquels ils s'inscrivent, les discours et positions des enseignants et orthophonistes algériens donnent à voir une certaine idée d'un "plurilinguisme ordinaire ", qui ne relève pas d'une prédéfinition de l'ordre, mais plutôt d'une prise en compte des personnes qui peuvent aider le professionnel à définir leurs besoins linguistiques. Nous pouvons alors rapprocher les positions des orthophonistes algériennes interrogées de l'idée de Jullien pour qui la « consistance » d'une société comme relève de sa « capacité d'écarts et de commun partagé » (2016, p. 75), les écarts poussant le « commun » au « travail », par le mouvement.

Enfin, en réaction aux injustices liées à la multiplication des situations scolaires dans lesquelles les enfants plurilingues, considérés comme " importunant » l'ordre scolaire (Ebersold et Armagnague, 2017), sont mécompris et "mésévalués " par des professionnels recrutés par une institution exclusive et excluante, nombreux sont les observateurs à considérer comme nécessaire et urgente une formation des enseignants aux problématiques du plurilinguisme, que ce dernier soit lié ou non à la migration (Armagnague et Bruneaud, 2016; Champalle et Galligani, 2015; Goi, 2015; Lebon-Eyquem et Robert, 2012; Rigoni, 2017). Dans les milieux de soin et d'éducation français où nous avons enquêté, nous avons pu identifier un certain nombre de problèmes, dont ceux liés aux conceptions de cet " autre étranger » - qui ne maîtrise pas le français mais maîtrise d'autres langues - et ceux du recours à des interprètes traducteurs qui pourraient faire avancer des situations bloquées. Si l'on conçoit culture et politique comme étant définies et nourries par le mouvement, l'écart et l'opposition, il conviendrait de reconnaître que la désignation et la dénomination de l' "autre " ne peuvent se faire sans convoquer une réflexion du " même » (Siblot, 2001, p. 14), tout comme l'« étranger » ne peut plus être une exception tolérée ou un désordre à ordonner, à l'école comme chez l'orthophoniste. Cassin écrit ainsi : "Les langues sont remises en mouvement [...], et la condition d'étranger, d'exilé, de déraciné, de barbare, d'autre, est une condition d'avant-garde » (2016, p. 220).

\section{Références bibliographiques}

Agamben, G. (1995). Moyens sans fins. Notes sur la politique. Paris : Payot.

Armagnague, M. et Bruneaud, J.-F. (2016). Introduction : Populations minorisées et justice scolaire : quelques enjeux pour les populations migrantes. Les cahiers de la LCD, 2(2), 11-19.

\footnotetext{
${ }^{21}$ Tous les enfants "allophones" ne sont pas plurilingues, mais s'ils sont nommés " allophones » par l'institution scolaire française, c'est qu'ils parlent une " autre " langue que "le " français. Étant scolarisés en et par « le » français, ils sont donc nécessairement des plurilingues en action et en devenir.
} 
Armagnague-Roucher, M. et Rigoni, I. (2016). Conduire une recherche sur la scolarisation d'élèves primo-migrants. Quelques enjeux et défis socioinstitutionnels, La nouvelle revue de l'adaptation et de la scolarisation, 75(3), 337-349.

Blanchet, P. (2012). La linguistique de terrain, méthode et théorie. Une approche ethnosociolinguistique de la complexité. Rennes : Presses universitaires de Rennes.

Blanchet, P. (2016). Discriminations : combattre la glottophobie. Paris : Textuel.

Brisset, C. et Leanza, Y. (2015). L'interprétariat en santé mentale à Montréal. Rhizome, 1(55), 20-27.

Bourdieu, P. (1982). Ce que parler veut dire. L'économie des échanges linguistiques. Paris : Fayard.

Bulot, T. et Blanchet, P. (2013). Introduction à la sociolinguistique. Pour l'étude des dynamiques de la langue française dans le monde. Paris : Éditions des archives contemporaines.

Canguilhem, G. (1999). Le normal et le pathologique. Paris : Presses universitaires de France.

Cassin, B. (2013). La Nostalgie. Quand donc est-on chez soi ? Paris : Autrement.

Cassin, B. (2016). Éloge de la traduction. Compliquer l'universel. Paris : Fayard.

Cassin, B. (2017). Présentation. Dans B. Cassin (dir.), Après Babel, traduire (p. 9-12). Arles/Marseille : Actes Sud.

Cattaneo, M.-L. et Scorzelli, K. (2017). Le rôle de la médiatrice linguistique et culturelle : I'expérience de la coopérative Crinali (Milan, Italie). L'autre, 18,(3), 388-395.

Caubet, D. (2004). Les Mots du Bled. Paris : L'Harmattan.

Champalle, A. et Galligani, S. (2015). L'enseignants face à la diversité linguistique et culturelle : difficultés et insécurités. Dans D.-L. Simon, C. Dompmartin-Normand, S. Galligani et M.-O. Maire Sandoz (dir.), Accueillir l'enfant et ses langues : rencontres pluridisciplinaires sur le terrain de l'école (p. 215-239). Paris : Riveneuves,.

Clément, P., Dreux, G., Vergne, F. et Laval, C. (2012). La nouvelle école capitaliste. Paris : La Découverte.

Ebersold, S. et Armagnague-Roucher, M. (2017). Importunité scolaire, orchestration de l'accessibilité et inégalités. Éducation et sociétés, 39(1), 137-152.

Fellag, M. (1995). Entretien avec Fellag. Dans D. Caubet (dir.), Les Mots du Bled (p. 31-37). Paris : L'Harmattan.

Fleury, C. (2015). Les irremplaçables. Paris : Gallimard.

Gobard, H. (1976). L'aliénation linguistique. Paris : Flammarion.

Gohard-Radenkovic, A. (2014). Peut-on enseigner des langues pensées comme "mauvaises langues" dans le processus d'intégration socioscolaire des élèves immigrés? D’apprentissages impensés à des pistes didactiques. Heteroglossia, 13, 1-30.

Goi, C. (2015). Des élèves venus d'ailleurs. Paris : Canopé Éditions.

Jaffredo, N. (2016). Vous avez dit orthophoniste ?. Dans Entre langue et parole, le métier d'orthophoniste (p. 185218). Toulouse : Erès.

Jullien, F. (2016). II n'y a pas d'identité culturelle. Paris : L'Herne.

Kebbas, M. et Kara-Abbes, A.-Y. (2010). Introduction. Dans P. Blanchet, M. Kebbas et A.-Y. Kara-Abbes (dir.), Influences et enjeux des contextes plurilingues sur les textes et les discours (p. 11-14). Limoges : LambertLucas.

Leanza, Y. (2017). Faire entrer le plurilinguisme dans les équipes cliniques. Analyse contextuelle du travail avec interprète. L'autre, 18(3), 272-281. 
Lebon-Eyquem, M. et Robert, C. (2012). La grande difficulté scolaire comme stigmate sociolinguistique. Dans M. Lebon-Eyquem, T. Bulot et G. Ledegen (dir.), Ségrégations, normes et discrimination(s). Sociolinguistique urbaine et migrance (p. 95-125). Paris : L'Harmattan.

Légifrance. (2016). Code pénal : Article 225-1 modifié par Loi n²016-1547 du 18 novembre 2016 [Internet]. https://www.legifrance.gouv.fr/affichCodeArticle.do?cidTexte=LEGITEXT000006070719\&idArticle=LEGIARTI 000006417828

Marchadour, M. (2018) Pluralité linguistique des individus dans l'éducation scolaire et le soin orthophonique. Algérie, France : enjeux politiques du " jeu » possible entre le même et l'autre (Thèse de doctorat, Université Rennes 2).

Mestre, C. (2017). Naissance de l'hospitalité dans la langue. L'autre, 18(3), 379-387.

Ministère de l'Éducation nationale (2012). Scolarisation des élèves. Circulaires n² 2012-141, 2012-142 et 2012-143 du 2 octobre 2012 [Internet]. Bulletin officiel de l'éducation nationale, 37, 11 octobre. http://www.education.gouv.fr/pid285/bulletin_officiel.html?pid_bo=26821

Ministère de l'Éducation nationale, Ministère de l'Enseignement supérieur, de la Recherche et de l'Innovation (2017). Repères et références statistiques. Enseignements, formation, recherche 2017 [Internet]. Paris : Ministère de I'Éducation nationale. http://cache.media.education.gouv.fr/file/2017/97/6/depp-RERS-2017maj-janv-2018_877976.pdf

Nathan, T. (1995). Les enfants de migrants et la politique scolaire (préface). Dans C. Mesmin (dir.), Psychothérapie des enfants de migrants (p. 11-22). Paris : Pensée Sauvage.

Noël, A. (2015). Création du BÉOCLER : Batterie d'Évaluation Orthophonique des Compétences Langagières des Enfants Réunionnais. De la conceptualisation à l'expérimentation (Thèse de doctorat, Université de La Réunion).

Rancière, J. (2000). Citoyenneté, culture et politique. Dans M. Elbaz et D. Helly (dir.), Mondialisation, citoyenneté et multiculturalisme (p. 55-68). Paris/Québec : L'Harmattan/Presses de l'université Laval.

Razafi, E. et Traisnel, C. (2017). Dire les minorités linguistiques en sciences sociales : les notions de " vitalité » et d' « allophone » dans les contextes canadien et français. Mots, 115, 111-126.

République algérienne (2016). Loi n 16-01 du 26 Joumada El Oula 1437 correspondant au 6 mars 2016 portant révision constitutionnelle [traduction française] [Internet]. Journal officiel de la République algérienne démocratique et populaire, 14, 1-38. https://www.joradp.dz/FTP/jo-francais/2016/F2016014.pdf

Ricœur, P. (2004). Sur la traduction. Paris : Bayard.

Rigoni, I. (2017). Accueillir les élèves migrants : dispositifs et interactions à l'école publique en France. Alterstice, 7(1), 39-50.

Rosenbaum, F. (2003). Clinique orthophonique et migration : la langue de la mère et l'œil du père. L'autre, 4(3), 411-427.

Rosenbaum, F. (2010). Les humiliations de l'exil. Les pathologies de la honte chez les enfants migrants. Paris : Fabert.

Rosenbaum, F. (2011). Les violences ou/et le racisme institutionnel dans un cadre psychopédagogique assimilationniste. Rééducation orthophonique, 247, 69-80.

Rosenbaum, F. (2012). L'orthophonie ethnoclinique à la croisée des cultures : les pièges et paradoxes des prises en charge des enfants de migrants. Dans P. Gatignol et S. Topouzkhanian (dir.), Bilinguisme et biculture : nouveaux défis? (p. 391-402). Paris : Ortho Édition.

Sayad, A. (1999). La double absence. Des illusions de l'émigré aux souffrances de l'immigré. Paris : Seuil. 
Sayad, A. (2014), L'immigration ou les paradoxes de l'altérité. La fabrication des identités culturelles. Paris : Raisons d'agir.

Siblot, P. (2001). De la dénomination à la nomination. Les dynamiques de la signifiance nominale et le propre du nom. Cahiers de praxématique, 36, 189-214.

Taleb-Ibrahimi, K. (2008). Enseignement de la langue arabe, méthodes, réformes et formation des maîtres en Algérie : état des lieux. Dans Le centenaire de l'agrégation d'arabe, Actes de la DGESCO (p. 139-143). Versailles : CRDP.

Varro, G. (1997). Les élèves “étrangers” dans les discours des institutions et des instituteurs. Langage et société, 80, 73-99.

Vincent, D. (2013). Langues nationales, variétés régionales et immigration. Les défis de l'école en français au Québec. Dans B. Falaize, C. Heimberg et O. Loubes (dir.), L'école et la nation (p. 235-246). Lyon : ENS Éditions. 